\title{
Mass Purging of Stale TCP Flows in Per-flow Monitoring Systems
}

\author{
Gunwoo Nam, Pushkar Patankar, George Kesidis, Chita R. Das \\ Dept. of Computer Science and Engineering, The Pennsylvania State University \\ Email: \{gnam, patankar, kesidis, das\}@ cse.psu.edu
}

\author{
Cetin Seren \\ Cisco Systems, Inc. \\ Email:cseren@cisco.com
}

\begin{abstract}
Timely deletion of a large number of stale sessions monitored by Internet routers, particularly in the presence of SYN floods, is critical to prevent flow table explosion. We investigate two frameworks for purging of stale sessions: "opportunistic" purging that employs a free-list of pointers to memory and "deterministic purging" involving logical swapping of a 1-bit flow enable and touch-bit vectors without requiring a free list. We compare the performance of our algorithms with a stateof-the-art algorithm, namely Finger-Compressed Filter (FCF). Our analysis using Internet traces shows that the deterministic purging, with no purging overhead, is ideal in that it reduces false positive and negative rates as compared to FCF by $52.5 \%$ and $59.2 \%$, when the table size is twice the average number of active flows.
\end{abstract}

\section{INTRODUCTION}

Flow-level measurement has become an important issue in networking devices to fulfill the current needs of congestion control, anomaly detection, QoS guarantees and usage-based accounting [1], [2]. To implement per-flow monitoring in high-end routers, more careful considerations are required for several reasons. First, table operations (flow look-up, update, and delete) should be performed at high-speed line rates. Second, flow records should be scalable to accommodate diverse applications of flow-based processing. Third, a flow table (or session table or connection records) should be capable of efficiently maintaining over 1 million flows [3].

Traditionally, flow tables have been implemented in DRAM [4], [5] and flow look-up is achieved by a tree-based search, hashing and Bloom filters techniques [6] [5], [7]. For highspeed line cards in the routers, implementation of the flow table using a fast but small SRAM for the flow table has been recently proposed in [1], [8]. These approaches hash the flow ID drawn directly from the packets to the address bus of the SRAM, which stores the flow state information. The hashing process should include methods to detect and correct for collisions, i.e., when different active TCP flows are hashed to the same location in the SRAM. Hence, the collision management process itself needs to be cognizant of which TCP flows are active and, thus, needs to account for flows when they are deemed inactive. Moreover, these techniques should allow various state information such as a packet (byte) counter and the timestamp of last packet received for that flow [5], [9], [10], be to maintained in the flow table, but any further

This work is supported by a Cisco Systems URP gift and by the NSF under grant CyberTrust 0524202 . extensions to it may be limited due to the smaller size of the SRAM.

Efficient flow table management has become critical to use the limited size of the SRAM. If the flow table is subjected to a large number of short-lived or abnormal flows, it may lead to enormous memory consumption and thereby considerable purging time overhead when a large number of flows time-out simultaneously. Also, this flow management mechanism itself could be vulnerable to malicious attacks, and any performance degradation of the router on which it is deployed could adversely affect the entire network connected to the Internet through it [11]. Attacks could impact flow monitoring indirectly or directly. A direct attack could be intended to soften the defense by, e.g., a preliminary SYN flood to preoccupy the monitoring defensive resources prior to the intended attack.

A stale flow might be replaced with recently arriving flow to the same RAM location, without employing periodic timeoutbased purging, by checking whether the existing flow has timed out. However, this requires a memory access to fetch the most recently touched time and a comparison with the current time to determine staleness. This process may have a high overhead, particularly during a SYN flood. In this paper, we address an efficient mass session purging issue of timedout flows, which has not been discussed extensively so far. Session purging needs to identify stale flows so that they can be removed and new flows can be instantiated. In a router, timed-out flows can be purged by using a global timer at the end of every timeout interval. However, this type of session purging also has significant time overhead. Purging operation should be as efficient as possible so that packet processing is not disrupted. This is especially challenging for a high speed router which is "barely" able to monitor per flow/packet state at line rate due to memory I/O limitations (only recently [5] argued that unsampled flow-level measurement itself was not sufficiently scalable, at that time, to be deployed in highend routers.). Similarly, naive replacement algorithms such as LRU may not work because it may be infeasible to maintain and update such timeout-ordered lists of active flows at high speed routers. It might be possible to prolong the effects of inefficient purging by allocating more than enough resources to maintain the flow table. However, if stale entries are rarely purged, over the course of time, the flow table, however large, will eventually be occupied with stale entries and new flows 
will not be instantiated in the flow table. To avoid flow table explosion, calibrating TCP session time-outs was explored in [12], [6]. Choice of time-out threshold may effect how many TCP sessions simultaneously expire, but there is an obvious trade-off in the selection of time-out thresholds: if too short, it is less vulnerable to SYN flooding but legitimate active flows could be falsely removed. If too large, the system becomes susceptible to SYN flooding. Managing such issues may be further complicated when the return data path of a session is not used, even temporarily, by the router monitoring the forward data path.

For mass purging of the deemed-stale TCP sessions in Internet routers, we start with a naive purging approach, called "opportunistic" purging. Opportunistic purging incorporates a combination of touch and enable bits along with a free list of pointers to available memory locations in the flow table. As long as time permits, stale flows are purged at the end of every timeout period by traversing all the flow entries. Thus, this scheme cannot guarantee massive purging of stale flows in a restricted time bound, which may detrimental realtime packet processing. Our experimental results indicate that the opportunistic purging suffers from conceivable purging overhead which is particularly problematic during periods where mass session deletions are required. To avoid massive purging overhead, we propose to use a separate memory (akin to one that could store the last-touched time) in a different way in the "deterministic" approach that has less overhead but basically results in a quantization of time for the purposes of determining time-out. "Deterministic purging" avoids massive purging overheads by logical swapping of touch and enable bits, without using a free list of pointers. The main idea of deterministic purging is the concept of invalidation, where outdated flows are invalidated using the touch/enable bits without actually deleting the corresponding flow entries in the flow table.

By using the deterministic purging we can easily keep track of the number of active flows. This quantity can be recomputed in a pipelined/parallel fashion during each phase. The number of actively flows is important when trying to detect "heavy hitter" flows (which are receiving more than their "fair share" bandwidth, a quantity that depends on the number of active flows, e.g., [13]) or when trying to detect denial-ofservice (DoS) flooding attacks that involve an abnormally large number of active flows (e.g., SYN flooding or Sybil/multipleidentity flooding at different levels of the protocol hierarchy).

The rest of this paper is organized as follows: In Section II, prior work is discussed. The opportunistic and deterministic purging mechanisms are described in Sections III and IV, respectively. Experimental results are given in Section V, followed by the conclusions in Section VI.

\section{RELATED WORK}

Before describing mass purging techniques, flow look-up and flow table implementations are investigated in this section. A flow is commonly defined using the (flow ID) time-proximal 5-tuple of IP and TCP headers [9]:

Flow ID : \{src ip, dst ip, src port, dst port, ip proto $\}$

Flow look-up can be achieved mostly by three ways: Hashing, Bloom Filter (BF) and Tenary Content-Addressable Memory (TCAM). To reduce flow table size, [4] and [8] use a hash of the "raw" flow ID and a hash bucket for hash-collision. The use of a hashed flow ID could result in a dramatically smaller entry size, but the (many-to-one) hash function may cause collisions. These hash table implementations have two drawbacks: First, they may increase the number of memory accesses through the use of bucket chaining to solve hash collisions. [7] pointed out this problem and proposed a fast look-up using a Shared-node Fast Hash Table (SFHT), a variant of the counting Bloom filter. Second, these techniques require storage of flow IDs (usually 104 bits) to check if there is a collision. To support up to 1 million concurrent flows, at least 104 Mbits $(104 \times 1 M)$ should be required for flow monitoring systems. Since BF techniques do not need to store full flow IDs, they have been proposed for fast flow lookup in small SRAMs. For example, [1] proposed a Fingerprint compressed Filter (FCF) to store 10-bits of compressed flow IDs via $d$-left hashing, which can reduce the total memory usage up to 20 Mbits. FCF consists of $d$ subtables and each hash bucket of the subtables has a fixed size without using pointers for fast lookup. When a new flow entry needs to be inserted, all $d$ possible buckets in subtables, by $d$ number of hash functions, are examined and the flow is placed in the bucket which has the fewest entries. Even though FCF can benefit from the use of the fast SRAM, but this also results in a limitation of scalability.

Apart from these software approaches, some packet classification approaches have used TCAMs for fast look-up in spite of its limited size, high price and high power consumption [14], [15], [16]. TCAM also can be considered an alternative for flow look-up. For a given technology, the TCAM' speed is determined by its depth and the width of the matching field. TCAMs on the order of $128 \mathrm{k}$ deep $\times 32$ bits wide are fast enough, for OC-192 forwarding purposes, which require $10 \times 10^{6}$ lookups per second [17]. With sufficient power and footprint in the linecard and on-chip and memory bandwidth headroom, multiple TCAMs can be combined to increase their depth (or width). Moreover, TCAMs thus configured do not suffer from collisions. Our proposed massive purging schemes could be adopted with any of these lookup mechanisms.

A few researchers have addressed the session purging issue in the flow table. Clearly, approaches that include updating complicated data structures as in tree based search [6], [18] at the start of every purging interval would be a non trivial task. FCF and two BF approaches, proposed in [1], use a single "touch" bit for time-out, which reduces lookup times for nonterminating flows. However, this touch bit is based on a bucket (a group of flows), rather than a single flow, i.e., a touch bit is set to 0 only if all the flows in the corresponding bucket have been inactive during the last timeout interval. This may increase the false positives (in terms of session purging), and 


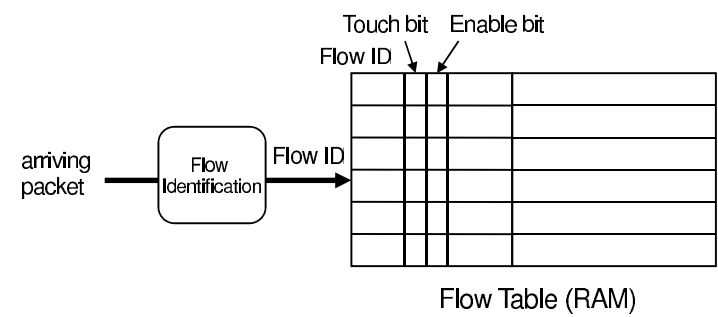

Fig. 1. Opportunistic purging

thereby, unnecessarily increase the number of active flows. As a flow measurement scheme, Adaptive NetFlow [5] was designed to be robust to flooding attacks by re-normalizing the flow entries based on the flow volume. When the number of flows exceeds a given threshold, it can reduce the flow entries by resampling with a new rate. It, however, can only report per-flow statistics which have been renormalized in the lowest sampling rate $r$ during a measurement interval.

\section{Opportunistic PURGing}

We start with a basic form of session purging, which we call "opportunistic". This purging scheme consists of a flow identification mechanism (see Figure 1), flow entries in a RAM and a free list of pointers. Each flow entry has a unique flow ID and an associated pointer to a high-speed RAM. The RAM is used to maintain the flow table, which includes flow IDs, the flows touch and enable bits, and additional flow information. The enable bit indicates whether the flow is active. The touch bit keeps track of the flow's activity in the current phase. The touch bit is set to 1 upon arrival of a packet with the same flow ID. If a router monitors TCP flows for the purpose of tracking only one out of the many possible states, high-speed on-chip SRAM can be possibly used, but we use a SDRAM in this paper because it provides a more scalable data structure. Also, a free-list of pointers to the RAM should be maintained to purge inactive flows' entries.

This flow monitor reacts to packet arrivals and the RAM/CPU perform back-end processing of the corresponding updated flow. In this setting, when a TCP packet arrives, the flow ID is extracted from the packet and a flow look-up is performed as follows:

- If the look-up succeeds, i.e., the flow is active, set the touch bit to 1 and update the flow's state in the RAM. If a RST or FIN packet is received, the corresponding flow entry is zeroed-out and the RAM pointer is moved to the free list.

- If the look-up fails, i.e., the flow is new, find an empty entry according to the existing structures, insert the flow entry in the flow table, and set its touch and enable bits to 1 .

Session purging at the end of every timeout phase $T_{\phi}$ involves a sequential traversal of all the flow entries and performing the following operations: if the touch bit is 0 and enable bit is 1, i.e., if the flow is timed out, the flow entry in the RAM is zeroed out and the pointer is moved to the free list. If the touch bit is 1 , it is reset to 0 .

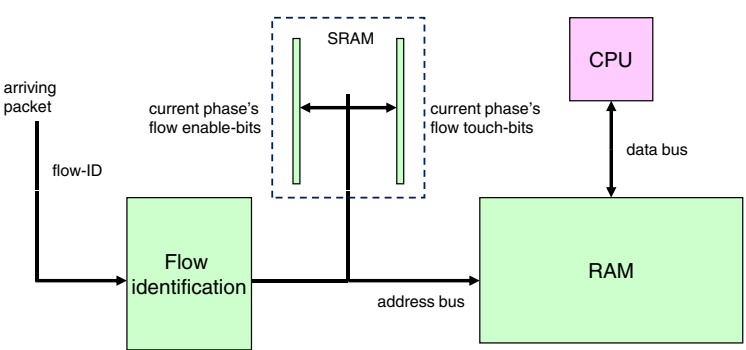

Fig. 2. Deterministic purging

State-dependent timeouts can be managed by reserving extra bits in the RAM for every flow and resetting the enable bit at the end of every timeout interval depending on the state of that flow. Though this mechanism can manage statedependent timeouts, it may introduce additional overhead to session purging. One problem of this purging method is that there could be excessive I/O operations when a large number of untouched flows need to be purged at the same time.

\section{Deterministic Purging}

Under the "deterministic" purging system, no free list of pointers to unoccupied or stale lines in the RAM is maintained. Instead, the flow table has a fixed size and all the empty entries are initialized in a specified way, say with entries of all zeroes. As shown in Figure 2, a flow enable and touchbits are maintained in a SRAM (called a touch-bit bitmap), separately from the flow table. In this system, outdated flows are invalidated using the flow enable-bits without actually removing the corresponding flow entries in the RAM. Also, the current touch-bit memory is used as the enable-bit memory in the next phase, i.e., swapping their function from phase to phase. Thus, it is possible to simultaneously delete all expired (untouched) flows upon conclusion of the current phase without incurring any purging time overhead. Since this mechanism does not require any memory I/O operations for session purging, it can be performed in a time-bound manner, hence, the name deterministic purging.

The basic premise of this approach is that the touch bits in one phase become the enable bits of the next phase and vice-versa i.e., the role of the flow touch and enable bits are logically swapped in a cycle of four phases. Consider the case, where a packet arrives and its flow has already been instantiated, so that the arriving packet toggles the touch bit to indicate that the flow is "touched" (see phase $n$ of Figure 3 ). At the end of phase $n$, the meaning of touch and enable bits are logically swapped. Arriving packets in phase $n+1$, set the touch bit to 0 . Hence, at the beginning of phase $n+2$, the enable bit is set to 0 , and the touch bit is 1 . Extending this logic for all the four phases as shown in Figure 3, it is easy to note that setting the enable bit to 1 in phases $n$ and $n+1$ indicates that the flow was active in those phases, while setting it to 1 in phases $n+2$ and $n+3$ indicates its inactiveness in those phases. If no packets are received in phase $n+2$, then at the beginning of phase $n+3$ the enable bit is set to 1 indicating that the flow is inactive. The invalidated flow entries can be reused for other new flows, without having to explicitly 


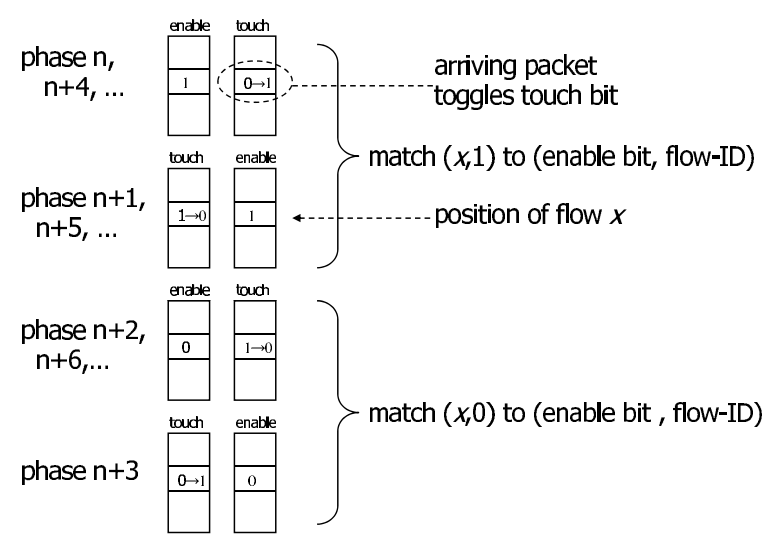

Fig. 3. An example of an active flow $\mathrm{x}$ in 4 consecutive phases

remove the previous flow entries. Therefore, the mechanism avoids writing a potentially enormous number of pointers to a free list memory at the conclusion of every phase.

If the flow is deemed active according to its enable bit, then the flow state (including the touch bit) is updated. If the flow is deemed inactive (i.e., the incoming packet belongs to a new flow), the corresponding flow entry in the flow table needs to be replaced with the new flow's information including the touch and enable bits.

\section{A. Implementation Issues}

Acting as an invalidation marker, the touch and enable bits should be accessed first to check whether the flow is active. Hence, touch and enable bits can be implemented using a bitmap, separated from the flow table. For flow lookup, it is feasible to employ a fixed-length hash table (or BF), e.g., perfect hashing or $d$-left hashing [1]. Or, we can use a TCAM in which flow IDs, touch bits and enable bits can be placed together.

Switching the meaning of touch and enable bits would require switching how an arriving packet's flow ID is augmented (i.e., alternate augmenting with 0 and 1 from phase to phase). For example, in the TCAM structure, flow lookup is performed by applying the packets augmented flow ID i.e., (flow ID:enable bit) to the TCAM's content bus. For example, in a phase $n$ where enabled 1 means that the flow is enabled, the touch bit's toggling from 0 to 1 indicates that the flow has been touched, and an arriving packet with a flow ID $\mathrm{x}$ is augmented with $1,(\mathrm{x}, 1)$. The polarity of the enable bit depends on the current phase. An empty TCAM entry is searched by applying (*:0) in phases $n$ and $n+1$, and $(*: 1)$ in phases $n+2$ and $n+3$ to its content bus.

\section{Performance Evaluation}

We conducted experiments using Internet traces, captured by LBL [19], NLALR [20] and MAWI [21]. The characteristics of the traces are given in Table I. We used a single timeout threshold $T_{\phi}$. In FCF, the number of subhashtables $(d)$ is set to 3 and the height of the bucket $(h)$ is 6 as described in [1]. The number of bucket $(b)$ is varied with the flow table size in our proposed purging schemes so that flow tables of FCF and our proposed schemes have the same size.

\begin{tabular}{|c||c|c|c|c|}
\hline Trace type & SMTP & HTTP & NZIX & B2006 \\
\hline \hline Record date & Sep. 05 & Sep. 05 & Jul. 00 & Jun. 06 \\
\hline Duration (min.) & 61 & 61 & 61 & 15 \\
\hline Total number of flows & 63,764 & 812,394 & 544,011 & 444,459 \\
\hline
\end{tabular}

TABLE I

INTERNET TRACE CHARACTERISTICS

\section{A. Objective Functions}

We use three objective metrics: purging time overhead, FPR and FNR to analyze our purging schemes and FCF.

1) Purging Time Overhead: To compare the memory access time overhead, we use the following parameters for memory access operations and access speed: $T_{l D R A M}$ and $T_{u D R A M}$ are the times for a look up and update (or insert and delete) in a DRAM, respectively, where $T_{u S R A M}$ and $T_{d S R A M}$ are the times for the SRAM update and delete, respectively. Based on these parameters, purging overheads are computed as follows:

- Purging time for the opportunistic purging,

$$
T_{d e l}=n \times T_{l D R A M}+k \times T_{d D R A M},
$$

where $n$ is average number of active flows and $k$ is average number of purged flows after every $T_{\phi}$.

- Purging time $T_{\text {del }}$ of the FCF scheme;

$$
T_{d e l}=d \times b \times T_{l S R A M}+k \times T_{d S R A M} .
$$

Recall that deterministic purging has no time overhead because purging is done by logically (not physically) swapping the enable bits and touch bits. We assume for the sake of simplicity that all RAM operations are performed in one cycle. We use SDRAM and SRAM access time as $8.1 \mathrm{~ns}$ [22] and $1.5 \mathrm{~ns}$ [23], respectively, for our evaluations.

2) FPR and FNR: The three approaches are also compared by observing whether flows that could have been deleted are not deleted (false-positive), and flows that should not be deleted are deleted (false-negative). To compare FPR and FNR, four parameters were measured at the end of every timeout phase $T_{\phi}$, assuming that $T_{c u r r}$ is the current phase-time and $T_{\text {next }}$ is the next phase-time, as follows:

- True positives (TP): Active flows at $T_{\text {curr }}$

- True negatives (TN): Normally terminated flows by receiving a RST/FIN packet + purged flows at $T_{\text {curr }}$

- False positives (FP): Purged flows at $T_{\text {next }}$

- False negatives (FN): Prematurely purged flows at $T_{c u r r}$

$$
F P R=\frac{F P}{F P+T N}, F N R=\frac{F N}{T P+F N} .
$$

Under deterministic purging and FCF, if there is no space in the flow table for new flows, either of the following can happen: 1) a newly arriving flow is dropped or 2) an existing flow, selected among the less recently active flows, is removed. For simplicity, we simply dropped the new flow, thereby adding a false negative, in our experiments. Therefore, when the number of active flows at any given instance exceeds the flow table size, there is a significant increase in false negatives. Opportunistic purging and deterministic purging showed the same performance in terms of FPR and FNR. So, in most 


\begin{tabular}{|c||c|c|c|c|c|}
\hline Scheme & Opp & Det & SFHT & FCF & ANF \\
\hline \hline $\begin{array}{c}\text { Memory } \\
\text { used }\end{array}$ & DRAM & $\begin{array}{c}\text { SRAM, } \\
\text { DRAM }\end{array}$ & $\begin{array}{c}\text { SRAM, } \\
\text { DRAM }\end{array}$ & SRAM & DRAM \\
\hline Latency & 2 & 3 & $j+n+1$ & $d+h+1$ & $2 p$ \\
\hline $\begin{array}{c}\text { Purging } \\
\text { type }\end{array}$ & Purging & $\begin{array}{c}\text { Logical } \\
\text { swapping }\end{array}$ & & $\begin{array}{c}\text { Time-based } \\
\text { deletion }\end{array}$ & $\begin{array}{c}\text { Renormal- } \\
\text { ization }\end{array}$ \\
\hline $\begin{array}{c}\text { Purging } \\
\text { time }\end{array}$ & $n+k$ & 0 & $n(1+k)$ & $d(b+k)$ & $m(n+k)$ \\
\hline
\end{tabular}

TABLE II

COMPARISON OF FLOW MONITORING ALGORITHMS

of the performance analysis, only the results of deterministic purging were plotted.

\section{B. Algorithm Comparison}

Table II shows the comparison of the proposed schemes with SFHT [7], FCF [1] and Adaptive NetFlow (ANF) [5] in terms of memory used, memory access time (latency) per table operation and worst case purging time. While opportunistic purging uses two memory accesses, a RAM lookup and update per flow table operation, the deterministic purging scheme uses three accesses, namely, a SRAM lookup, a SRAM update and a RAM update. The SFHT technique first accesses $j$ locations of the Bloom Filter by using number of hash functions $(j)$. It then takes up to $n$ accesses, in the worst case, for a hash bucket search to insert (or update) an entry. [7] did not describe any specific purging schemes for SFHT, though it clearly incurs purging overhead in linear time, $O(n)$, if a timeout based purging is assumed. As the height of a bucket $h$ is fixed, memory accesses in FCF are faster than under SFHT, even though flow lookup is required for $d$ different subhashtables. The FCF's purging overhead is proportional to $d$ and $b$ since purging is based on a bucket (group of flows).

ANF, designed for off-line measurement, reduces memory accesses per operation using flow sampling. The purging overhead of ANF is dependent on the number of renormalizations $m$. It is not trivial to use this renormalization scheme in a real-time monitoring system, because of the overheads caused by time required for renormalizations and flow entries' synchronization. Therefore, we implemented only FCF, which shows the best purging time among these state of the art approaches, for purpose of comparison.

\section{Design Trade-offs}

1) Impact of Timeout Threshold $\left(T_{\phi}\right)$ : Figure 4 shows the average number of active flows, average number of purged flows and FNR using deterministic purging for the four traces. The flow table was set to a conceivable size of 200,000 entries and $T_{\phi}$ was varied $\left(30,60,120\right.$, and 300 seconds). As $T_{\phi}$ was increased, average number of active flows increased as expected, while FNR decreased. Consistent with the difficulty of setting up a time-out threshold as discussed in [6], [12], Figure 4(c) shows that a certain small FNR level was present under deterministic purging. However, FNR was less than 5\% even with timeout thresholds of 60 or 120 seconds.

Figure 4(b) depicts that as $T_{\phi}$ was increased, the average number of purged flows also increased, but the total number

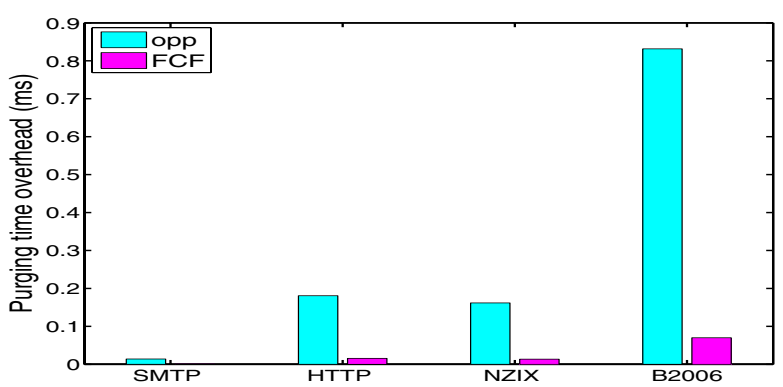

Fig. 6. Purging overhead ( $T_{\phi}=120$ seconds)

of purged flows at the end of a specific time period remained approximately the same irrespective of the timeout thresholds. This indicates that for larger (respectively, smaller) timeout thresholds, the flows that needed to be purged were kept in the flow table for a longer (respectively, shorter) duration.

2) Impact of Flow Table Size: Figure 5 shows the comparisons between deterministic purging and FCF for the HTTP trace as a function of the flow table size. The timeout threshold was set to 120 seconds. Note that FNR is much more significant because a higher FNR value indicates that active flows are not managed properly in the flow table, while a higher FPR indicates unnecessary occupancy of timed-out flows in the flow table.

Figures 5(a) and 5(b) show that FCF estimated a larger number of active flows and higher FPR due to the FCF's inefficient bucket-level purging described Section II. From Figure 5(a), we can see that the utilization of deterministic purging whose flow table size is $3 X$ was $33 \%$, where $X$ is average number of active flows. However, the utilization of FCF under the same size was $65 \%$ because stale flows were unnecessarily kept. It caused bucket overflow so that a new flow could not be added. As a result, we can see that the FNR of FCF was significantly high until the flow table became $3 X$ from Figure 5(c). This observation implies that FCF required much more flow memory than real number of concurrent flows. Note that when the flow table size was $3 X$ in Figure 5(c), the FNR of FCF was similar to that of deterministic purging just because the estimated number of active flows under FCF were much larger than those of the deterministic purging, as shown in 5(a). The results of the other three traces were similar, thus omitted here.

\section{Purging Time Overhead}

Figure 6 plots the time overhead of opportunistic purging and FCF based on the equations (2) and (3). We obtained the value of $n$ and $k$ using experiments performed in Section V-C. Figure 6 indicates that FCF showed a smaller overhead than opportunistic purging because of the use of fast SRAM and a smaller number of purged flows than our schemes. Deterministic purging incurs no purging overhead as discussed before. Considering that an OC-192 linecard can process 50,000 packets $/ \mathrm{ms}$, purging time overhead, e.g., $0.83 \mathrm{~ms}$ in opportunistic purging for the B2006 trace, may result in flow monitoring downtimes. Time overhead of opportunistic purging in a realistic scenario, which might contain quite a 


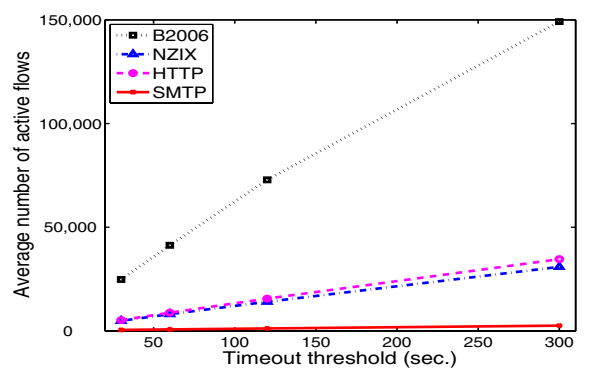

(a) Avg. number of active flows

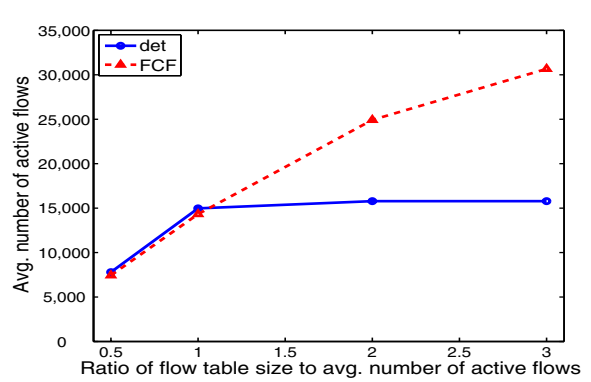

(a) Avg. number of active flows

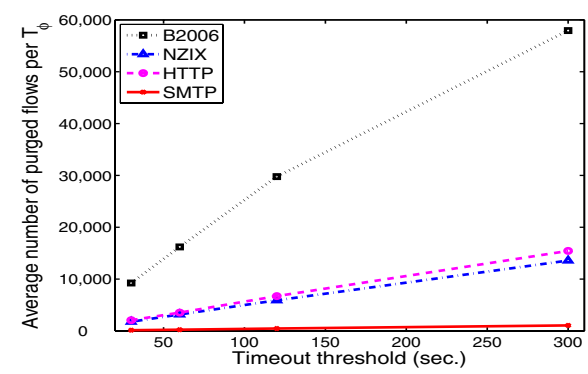

(b) Avg. number of purged flows per $T_{\phi}$

Fig. 4. Deterministic purging with varying $T_{\phi}$

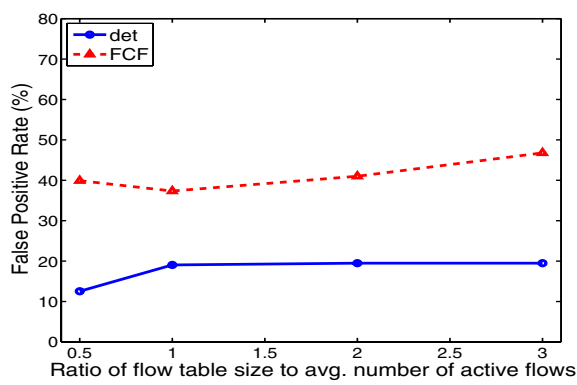

(b) Average FPR

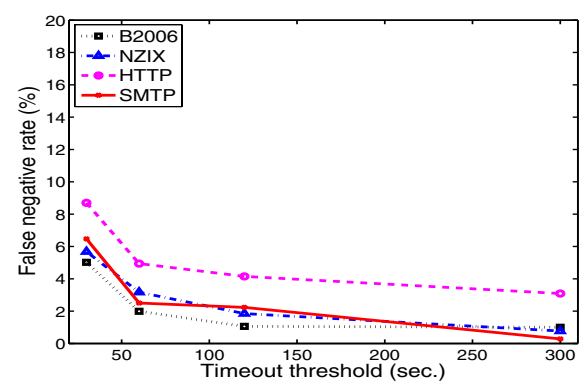

(c) Average FNR

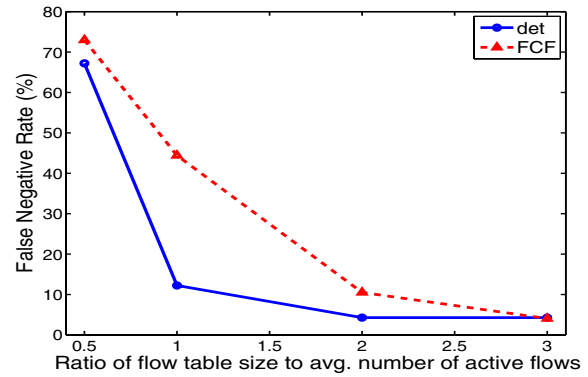

(c) Average FNR

Fig. 5. Comparing deterministic purging and FCF with varying table size (HTTP trace, $T_{\phi}=120$ seconds)

large number of concurrently active flows (thereby increasing the number of purged flows at every timeout interval), will be significantly higher. y higher.

\section{FUTURE WORK}

We plan to extend our purging approaches to accommodate several state dependent time-out multiple-phase thresholds per flow. Hash collisions between two active flows motivates the need for a collision recovery mechanism, e.g., [24]. For example, to protect a spurious SYN packet overwriting a legitimate active flow, we can store the last two touch bits and assume that the flow entry is not completely deleted upon arrival of the new SYN packet. While one of the touch bits is modified by the SYN packet, this can be corrected when the next packet of the original flow arrives. We also plan to extend our purging frameworks to monitor long-duration UDP sessions.

\section{REFERENCES}

[1] F. Bonomi, M. Mitzenmacher, R. Panigrahy, S. Singh, and G. Varghese, "Beyond bloom filters: from approximate membership checks to approximate state machines," in Proc. ACM SIGCOMM, 2006.

[2] "Cisco NetFlow," http://www.cisco.com/warp/public/732/Tech/netflow.

[3] K. Levchenko, R. Paturi, and G. Varghese, "On the difficulty of scalably detecting network attacks," in Proc. ACM CCS, 2004, pp. 12-20.

[4] D. V. Schuehler, J. Moscola, and J. W. Lockwood, "Architecture for a Hardware-Based, TCP/IP Content-Processing System," IEEE Micro, vol. 24, no. 1, pp. 62-69, 2004.

[5] C. Estan, K. Keys, D. Moore, and G. Varghese, "Building a better NetFlow," in Proc. ACM SIGCOMM, 2004.

[6] X. Li, Z. Ji, and M. Hu, "Session Table Architecture for Defending SYN Flood Attack," in Proc. ICICS, 2005.

[7] H. Song, S. Dharmapurikar, J. Turner, and J. Lockwood, "Fast Hash Table Lookup Using Extended Bloom Filter: An Aid to Network Processing," in Proc. ACM SIGCOMM, 2005.
[8] S. Yoon, B. koo Kim, J. Oh, and J. Jang, "High Performance Session State Management Scheme for Stateful Packet Inspection," in Proc. APNOMS, 2007, pp. 591-594.

[9] N. Duffield, C. Lund, and M. Thorup, "Estimating flow distributions from sampled flow statistics," in Proc. ACM SIGCOMM, 2003.

[10] IETF Working Group, "Internet Protocol Flow Information eXport (IPFIX)," http://net.doit.wisc.edu/ipfix/, 2006.

[11] S. Dharmapurikar and V. Paxson, "Robust TCP stream reassembly in the presence of adversaries," in Proc. USENIX SSYM'05, 2005.

[12] H. Kim, J.-H. Kim, I. Kang, and S. Bahk, "Preventing Session Table Explosion in Packet Inspection Computers," IEEE Trans. Computers, vol. 54, no. 2, pp. 238-240, 2005.

[13] S. Yi, X. Deng, G. Kesidis, and C. R. Das, "A dynamic quarantine scheme for controlling unresponsive tcp sessions," Telecommunication Systems, vol. 37, no. 4, pp. 169-189, 2008.

[14] F. Zane, G. J. Narlikar, and A. Basu, "CoolCAMs: Power-Efficient TCAMs for Forwarding Engines," in Proc. IEEE INFOCOM, 2003.

[15] C. R. Meiners, A. X. Liu, and E. Torng, "TCAM Razor: A Systematic Approach Towards Minimizing Packet Classifiers in TCAMs," in Proc. IEEE ICNP, 2007.

[16] K. Lakshminarayanan, A. Rangarajan, and S. Venkatachary, "Algorithms for advanced packet classification with ternary CAMs," in Proc. ACM SIGCOMM, 2005.

[17] P. Gupta, S. Lin, and N. McKeown, "Routing lookups in hardware at memory access speeds," in Proc. IEEE INFOCOM, 1998.

[18] X. Li, Z.-Z. Ji, and M.-Z. Hu, "Stateful Inspection Firewall Session Table Processing," in Proc. International Conference on Information Technology: Coding and Computing (ITCC), 2005, pp. 615-620.

[19] "LBNL/ICSI Enterprise Tracing Project," http://www.icir.org/enterprisetracing/.

[20] "The Passive Measurement and Analysis projects," http://pma.nlanr.net/.

[21] "WIDE traffic archive," http://tracer.csl.sony.co.jp/.

[22] H. Fujisawa, S. Kubouchi, K. Kuroki, N. Nishioka, Y. Riho, H. Noda, I. Fujii, H. Yoko, R. Takishita, T. Ito, H. Tanaka, and M. Nakamura, "An 8.1-ns Column-Access 1.6-Gb/s/pin DDR3 SDRAM With an 8:4 Multiplexed Data-Transfer Scheme," IEEE Journal of Solid-State Circuits, vol. 40, no. 1, pp. 201-209, Jan. 2007.

[23] "Packet buffer," http://yuba.stanford.edu/or/.

[24] J. Xu and M. Singhal, "Cost-Effective Flow Table Designs for HighSpeed Routers: Architecture and Performance Evaluation," IEEE Trans. Comput., vol. 51, no. 9, pp. 1089-1099, 2002. 